\title{
BMJ
}

\section{Conventional versus automated measurement of blood pressure in primary care patients with systolic hypertension: randomised parallel design controlled trial}

\author{
Martin G Myers, professor of medicine, ${ }^{1}$ Marshall Godwin, professor of medicine, ${ }^{2}$ Martin Dawes, professor of \\ medicine, ${ }^{3}$ Alexander Kiss, assistant professor, ${ }^{4}$ Sheldon W Tobe, associate professor of medicine, ${ }^{5}$ F Curry \\ Grant, director, ${ }^{6}$ Janusz Kaczorowski, professor of medicine ${ }^{7}$
}

${ }^{1}$ Schulich Heart Centre, Department of Medicine, University of Toronto, Toronto, ON, Canada

${ }^{2}$ Department of Family Medicine, Memorial University of

Newfoundland, St John's, NF,

Canada

${ }^{3}$ Department of Family Medicine, McGill University, Montreal, QC,

Canada

${ }^{4}$ Department of Research Design and Biostatistics, Sunnybrook Health Sciences Centre, Toronto

${ }^{5}$ Department of Medicine, University of Toronto

${ }^{6}$ Stroke Prevention Clinic, Quinte Health Care, Belleville, ON,

Canada.

${ }^{7}$ Department of Family Practice, University of British Columbia, Vancouver, Canada

Correspondence to: M Myers, Schulich Heart Centre, Division of Cardiology, Sunnybrook Health Sciences Centre, A-202, 2075 Bayview Avenue, Toronto, ON, Canada M4N 3M5 martin.myers@sunnybrook.ca

Cite this as: BMJ 2011;342:d286 doi:10.1136/bmj.d286

\section{ABSTRACT}

Objective To compare the quality and accuracy of manual office blood pressure and automated office blood pressure using the awake ambulatory blood pressure as a gold standard.

Design Multi-site cluster randomised controlled trial.

Setting Primary care practices in five cities in eastern Canada.

Participants 555 patients with systolic hypertension and no serious comorbidities under the care of 88 primary care physicians in 67 practices in the community. Interventions Practices were randomly allocated to either ongoing use of manual office blood pressure (control group) or automated office blood pressure (intervention group) using the BpTRU device. The last routine manual office blood pressure $(\mathrm{mm} \mathrm{Hg}$ ) was obtained from each patient's medical record before enrolment. Office blood pressure readings were compared before and after enrolment in the intervention and control groups; all readings were also compared with the awake ambulatory blood pressure.

Main outcome measure Difference in systolic blood pressure between awake ambulatory blood pressure minus automated office blood pressure and awake ambulatory blood pressure minus manual office blood pressure.

Results Cluster randomisation allocated 31 practices (252 patients) to manual office blood pressure and 36 practices (303 patients) to automated office blood pressure measurement. The most recent routine manual office blood pressure (149.5 (SD 10.8)/81.4 (8.3)) was higher than automated office blood pressure (135.6 (17.3)/77.7 (10.9)) ( $\mathrm{P}<0.001)$. In the control group, routine manual office blood pressure before enrolment (149.9 (10.7)/81.8 (8.5)) was reduced to 141.4 (14.6)/80.2 (9.5) after enrolment $(P<0.001 / P=0.01)$, but the reduction in the intervention group from manual office to automated office blood pressure was significantly greater $(P<0.001 / P=0.02)$. On the first study visit after enrolment, the estimated mean difference for the intervention group between the awake ambulatory systolic/diastolic blood pressure and automated office blood pressure ( -2.3 (95\% confidence interval -0.31 to -4.3$) /-3.3(-2.7$ to -4.4$))$ was less $(P=0.006 / P=0.26)$ than the difference in the control group between the awake ambulatory blood pressure and the manual office blood pressure $(-6.5(-4.3$ to -8.6$) /-4.3$ $(-2.9$ to -5.8$))$. Systolic/diastolic automated office blood pressure showed a stronger $(\mathrm{P}<0.001)$ within group correlation $(r=0.34 / r=0.56)$ with awake ambulatory blood pressure after enrolment compared with manual office blood pressure versus awake ambulatory blood pressure before enrolment $(r=0.10 / r=0.40)$; the mean difference in $r$ was 0.24 (0.12 to 0.36$) / 0.16$ (0.07 to 0.25$)$ ). The between group correlation comparing diastolic automated office blood pressure and awake ambulatory blood pressure $(r=0.56)$ was stronger $(P<0.001)$ than that for manual office blood pressure versus awake ambulatory blood pressure $(r=0.30)$; the mean difference in $r$ was 0.26 (0.09 to 0.41). Digit preference with readings ending in zero was substantially reduced by use of automated office blood pressure.

Conclusion In compliant, otherwise healthy, primary care patients with systolic hypertension, introduction of automated office blood pressure measurement into routine primary care significantly reduced the white coat response compared with the ongoing use of manual office blood pressure measurement. The quality and accuracy of automated office blood pressure in relation to the awake ambulatory blood pressure was also significantly better when compared with manual office blood pressure. Trial registration Clinical trials NCT 00214053.

\section{INTRODUCTION}

Despite intensive efforts to promote proper techniques for the measurement of blood pressure, widespread concern about the quality and accuracy of blood pressure measurement in "real life" clinical settings continues. ${ }^{12}$ Studies from routine clinical practice often report imprecise and inconsistent manual office blood pressure readings owing to poor measurement technique, patient-physician interaction such as conversation during readings, and failure to minimise patient related factors such as anxiety. ${ }^{34}$ Recent studies suggest that an accurate office blood pressure reading 
requires at least 14 minutes, including a period of rest before the first measurement. ${ }^{56}$ The likelihood of such careful adherence to protocols for blood pressure measurement in routine, community based, office practice would seem to be low.

In recognising these concerns about manual office blood pressure measurement, some experts in hypertension have recently recommended a more limited role for such readings. Proposals for improved assessment of blood pressure status include greater reliance on home and 24 hour ambulatory blood pressure monitoring. ${ }^{78}$ Not only do out of office readings eliminate increased readings associated with conventional manual office blood pressure measurement (the white coat response), they are also stronger predictors of future cardiovascular events. ${ }^{9}{ }^{10}$ Detection and elimination of the white coat response also reduces the number of patients who receive unnecessary drug treatment. ${ }^{11}$

Although the evidence supporting greater use of home and 24 hour ambulatory blood pressure monitoring is substantial, to consider abandoning office blood pressure measurement because of the deficiencies associated with conventional manual office readings is both premature and unwise. Recent advances in the development of automated sphygmomanometers provide a third option for accurate assessment of blood pressure status: automated office blood pressure measurement. The use of independently validated, fully automated sphygmomanometers designed for professional use reduces or eliminates many of the factors contributing to imprecise blood pressure readings in routine clinical practice. ${ }^{12-14}$ Self measurement with the patient resting alone in a quiet examining room makes it possible to eliminate patient-observer interaction such as conversation, an important cause of the white coat response. ${ }^{1516}$ The absence of a health professional during blood pressure measurement also seems to reduce the anxiety that many patients experience during visits to their doctor. ${ }^{17} 18$

We designed the Conventional versus Automated Measurement of Blood pressure in the Office (CAMBO) trial to evaluate the effect of automated versus manual office blood pressure measurement on the management of hypertensive patients in routine, community based, clinical practice over a two year period. Patients with predominantly systolic hypertension were eligible for the study, as the white coat response is associated with a disproportionate increase in systolic blood pressure. ${ }^{2}$ This report presents findings from the first phase of CAMBO, examining whether automated office blood pressure can reduce the white coat response and improve on the quality and accuracy of manual office blood pressure by producing readings that are similar to and more strongly correlated with the awake ambulatory blood pressure.

\section{METHODS}

Study design

We invited community based family physicians in five Canadian cities who were using manual office blood pressure in their clinical practice to participate in the study. Physicians' practices (sites) consisted of either one family physician or a group of two or three physicians sharing the same office space. To reduce potential contamination, we used a cluster randomisation design in which we randomised sites rather than individual patients to either manual office blood pressure with continuing use of manual sphygmomanometry (control group) or management of the study patients with automated office blood pressure (intervention group) using the BpTRU device (BpTRU Medical Devices, Coquitlam, BC, Canada). We stratified randomisation to management with automated or manual office blood pressure measurement by city to minimise possible regional differences in blood pressure measurement and management of hypertension arising from any imbalance between the groups. We set a target of 10 patients for each site, with a minimum of five patients. We allowed up to 15 patients for practices in which two or three physicians at one site were randomised as a single cluster.

\section{Recruitment of patients}

We considered patients with a hypertension code on billing forms to be eligible for the study if they satisfied the initial screening criteria, including age over 45 years, no serious coexisting illness limiting participation, no history of non-compliance, not treated for diabetes mellitus, serum creatinine less than twice normal, and not using or intending to use home blood pressure measurements. We required untreated patients to have a systolic blood pressure of at least $160 \mathrm{~mm} \mathrm{Hg}$ and diastolic blood pressure below $95 \mathrm{~mm} \mathrm{Hg}$ on their most recent pre-study visit, as recorded in their medical chart. Patients already receiving antihypertensive treatment were eligible if their systolic blood pressure was at least $140 \mathrm{~mm} \mathrm{Hg}$ and their diastolic pressure was below $90 \mathrm{~mm} \mathrm{Hg}$. Potential participants were sent a letter from the office of their family physician asking if they would be interested in participating in CAMBO. Patients who agreed to attend for a baseline visit were seen by a study nurse, who explained the details of the study and verified that the patients satisfied all of the inclusion criteria. Eligible patients who agreed to participate then had 24 hour ambulatory blood pressure monitoring before the next scheduled follow-up visit with their regular physician. The last routine manual office blood pressure recorded on the visit immediately before entry into the study was documented. We developed an intensity score using arbitrary units for each antihypertensive drug, to assess the amount of antihypertensive drugs being taken by each patient at baseline and during subsequent visits (for example, 1 unit for hydrochlorothiazide $=12.5 \mathrm{mg}$, atenolol $=25 \mathrm{mg}$, amlodipine $=2.5 \mathrm{mg}$ ). We defined excess alcohol consumption as 14 or more standard alcoholic beverages a week for men and nine or more beverages a week for women.

\section{Blood pressure measurement}

The BpTRU is a fully automated sphygmomanometer that records blood pressure by the oscillometric 


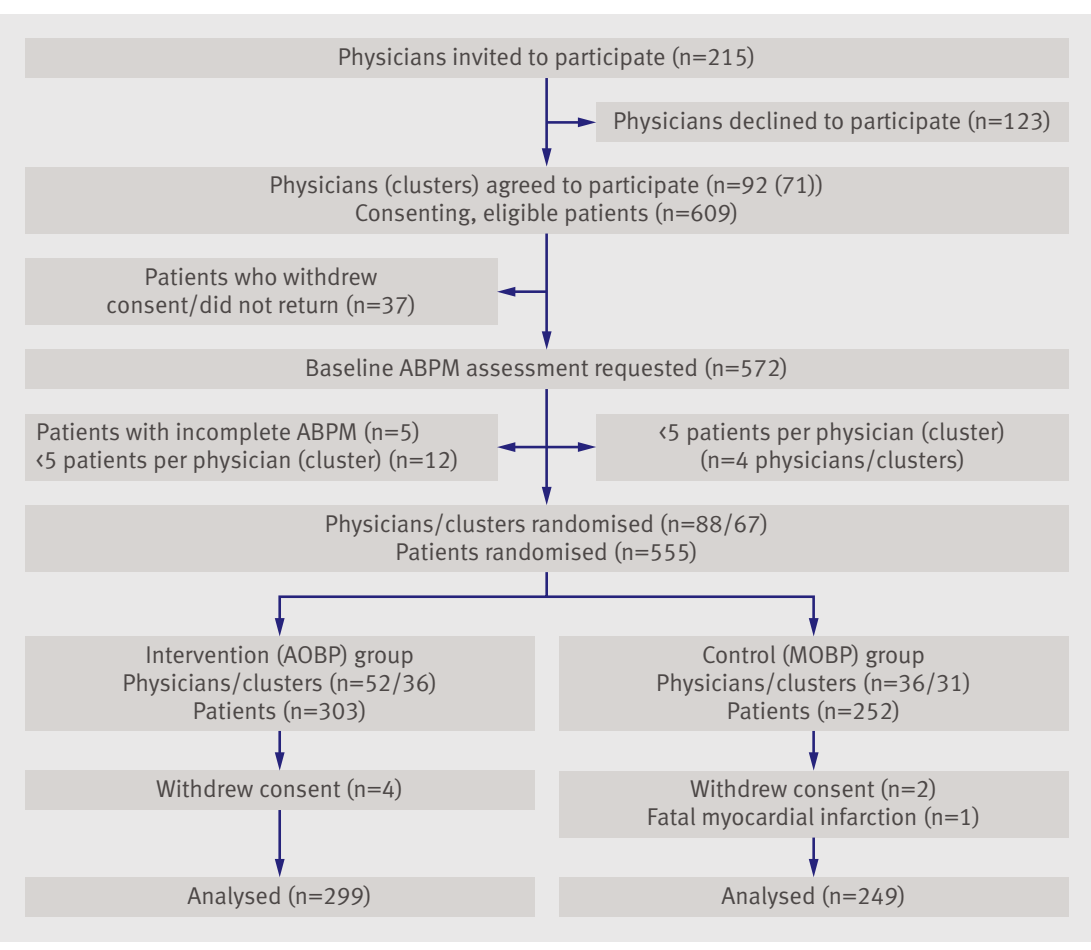

Fig 1 Flow diagram showing recruitment of family physicians and patients into trial. $A B P M=24$ hour ambulatory blood pressure monitoring; $A O B P=$ automated office blood pressure; $M O B P=$ manual office blood pressure at 30 minute intervals during the night. We then calculated the mean awake ambulatory blood pressure according to the actual awake period as recorded in each patient's diary, where time asleep was noted.

\section{Data analysis}

We calculated descriptive statistics for variables of interest. We report continuous variables as mean and standard deviation and show differences between mean values with confidence intervals. This analysis focuses on baseline comparisons of blood pressure readings between participants randomised to the two study arms using manual office blood pressure, automated office blood pressure, and 24 hour ambulatory blood pressure monitoring. We use Bland-Altman scatter plots to show differences between blood pressure measurements, ${ }^{21}$ which we evaluated by using repeated measures analysis of variance models. The models took into account the correlated nature of the data and the potential data dependency associated with the cluster design.

We assessed differences in coefficients of correlation between comparisons of blood pressure readings by using independent correlation coefficients for between group comparisons and correlated coefficients for within group comparisons. We used $\chi^{2}$ tests to evaluate differences between groups in the proportion of patients showing digit preference (rounding off blood pressure readings to the nearest zero value). Applying the method of Donner et al, ${ }^{22}$ the study was powered for the primary outcome measure to detect a difference of $5 \mathrm{~mm} \mathrm{Hg}$ between groups (difference in awake ambulatory systolic blood pressure minus manual office blood pressure versus difference in awake ambulatory systolic blood pressure minus automated office blood pressure), with $90 \%$ power at $\alpha=0.05$, a hypothesised intracluster correlation of 0.07 , and a $20 \%$ attrition rate. The resulting estimated sample size was 276 participants per group. We used SAS version 9.1 for all analyses.

\section{RESULTS}

We recruited 572 patients from primary care practices in four regions in central and eastern Canada: Belleville (Ontario), Brantford (Ontario), Montreal (Quebec), and St John's/Corner Brook (Newfoundland), as shown in figure 1 . We excluded 12 patients from participation because fewer than five patients were recruited from each of their physicians' practices. Five other patients declined to have 24 hour ambulatory blood pressure monitoring after having signed the consent form. The cluster randomisation process allocated 303 patients to management of their hypertension with automated office blood pressure (intervention group) and 252 patients to ongoing manual office blood pressure measurement (control group). The intervention group consisted of 36 practices (52 physicians), compared with 31 practices (36 physicians) in the control group. The imbalance in the number of patients in each group was partly due to more sites with multiple physicians being randomised to 
automated office blood pressure. In addition, randomisation was stratified by the four regions (Brantford, Belleville, Montreal, and St John's/Corner Brook) and, by chance, this resulted in more sites being allocated to the intervention group. After randomisation, four patients in the intervention group and two patients in the control group withdrew consent before attending the first within study office visit. Another patient in the control group had a fatal myocardial infarction before his return office visit.

No significant differences existed in the characteristics of the patients randomised to the two groups (table 1). Routine manual office blood pressure readings taken during the visit immediately before enrolment into the study and the mean awake ambulatory blood pressure at baseline were similar in the two groups (table 2).

With respect to the primary outcome measure, the mean estimated difference between the mean awake ambulatory systolic/diastolic blood pressure and the automated office blood pressure in the intervention group at the first office visit after enrolment was -2.3 (95\% confidence interval -0.3 to -4.3$) /-3.3$ ( -2.2 to $-4.4)(\mathrm{P}=0.02 / \mathrm{P}<0.001)$. The corresponding difference between the awake ambulatory blood pressure and manual office blood pressure for the first post-enrolment office visit in the control group was $-6.5(-4.3$ to -8.6$) /-4.3 \quad(-2.9$ to -5.8$) \quad(\mathrm{P}<0.001 / \mathrm{P}<0.001)$ (table 2). The mean estimated difference for systolic manual office blood pressure $(-6.5)$ was significantly greater $(\mathrm{P}=0.006)$ than that for systolic automated office blood pressure (-2.3). The difference for diastolic manual office blood pressure was not significant $(\mathrm{P}=0.26)$.

Both groups showed a fall in mean office blood pressure between the most recently recorded pre-study routine office visit and the first visit to the physician after enrolment in the study (table 2). Mean blood pressure in the automated office blood pressure group was reduced by 13.9 (11.8 to 16.1$) / 3.7$ (2.5 to 4.8$) \mathrm{mm} \mathrm{Hg}$, which was more $(\mathrm{P}<0.001 / \mathrm{P}=0.02)$ than for the manual office blood pressure patients $(8.5$ (6.5 to 10.4$) / 1.6(0.4$ to 2.8$) \mathrm{mm} \mathrm{Hg}$ ). None of the patients changed antihypertensive drug treatment between the last routine

Table 1/Characteristics of patients who completed baseline period and were randomised to intervention group (automated office blood pressure) or usual care group (continued manual office blood pressure) for management of hypertension. Values are numbers (percentages) unless stated otherwise

\begin{tabular}{|c|c|c|}
\hline Characteristic & $\begin{array}{l}\text { Automated office blood } \\
\text { pressure }(n=303)\end{array}$ & $\begin{array}{l}\text { Conventional manual office } \\
\text { blood pressure }(n=252)\end{array}$ \\
\hline Male sex & $107(35)$ & $103(41)$ \\
\hline Mean (range) age (years) & $65(45-90)$ & $65(46-84)$ \\
\hline Mean (SD) duration of hypertension (years) & $9.2(8.9)$ & $9.6(10.4)$ \\
\hline Not receiving antihypertensive treatment & $13(4)$ & $12(5)$ \\
\hline $\begin{array}{l}\text { Mean (SD) intensity of antihypertensive drug } \\
\text { treatment (units) }\end{array}$ & $3.7(2.3)$ & $3.5(2.2)$ \\
\hline Cigarette smokers & 39 (13) & $37(15)$ \\
\hline Excess alcohol use & $21(7)$ & $13(5)$ \\
\hline
\end{tabular}

office visit and the first visit to the office after the 24 hour ambulatory blood pressure monitoring.

Routine manual office systolic blood pressure readings in both groups taken before participation in the study correlated poorly with the mean awake ambulatory blood pressure (table 3 ). For patients in the intervention group, the within group correlations between awake ambulatory blood pressure and systolic/diastolic automated office blood pressure at the first office visit after enrolment in the study were significantly higher than those for the ambulatory blood pressure versus the last routine manual office blood pressure before entry into the study $(r=0.34$ versus $r=0.10$ (95\% confidence interval for change 0.12 to 0.36 ), $\mathrm{P}<0.001 / r=0.56$ versus $r=0.40 \quad(0.07$ to 0.25$)$, $\mathrm{P}<0.001)$. For the control group, we saw both positive and negative changes in correlation coefficients between the manual office systolic/diastolic blood pressure readings and the awake ambulatory blood pressure from before to after enrolment in the study $(r=0.04$ versus $r=0.22$ ( 0.02 to 0.32$), \mathrm{P}=0.03 / r=0.42$ versus $r=0.30(0.0,-0.01$ to 0.24$), \mathrm{P}=0.08)$. The between group correlation between diastolic automated office blood pressure and awake ambulatory blood pressure $(r=0.56)$ was stronger $(\mathrm{P}=<0.001)$ than that for manual office blood pressure versus awake ambulatory blood pressure $(r=0.30)$; the mean difference in $r$ was 0.26 (0.09 to 0.41$)$.

We plotted the mean awake ambulatory and office systolic blood pressure readings against the difference between these readings by using the Bland-Altman scatter plot format (fig 2). ${ }^{21}$ Routine mean pre-entry manual office blood pressure readings (top panels) showed a marked positive bias in the intervention group (16.3 (2 SD -14.4-46.9)) and control group (14.9 (-17.5-47.3)), whereas the automated office blood pressure readings (bottom left panel) showed minimal bias $(2.3(-31.9-36.6))$, reflecting less of a white coat response. Manual office blood pressure readings taken on the first office visit after enrolment (bottom right panel) showed a moderate degree of bias (6.5 (-27.6-40.5)), consistent with a partial fall in blood pressure compared with routine manual office blood pressure readings recorded before enrolment.

Digit preference, with manual office blood pressure readings being rounded off to the nearest zero, was common in both the automated and manual office blood pressure groups at the last routine office visit before enrolment. For automated office blood pressure patients, the number of individual readings that showed digit preference for systolic/diastolic blood pressure at this visit $(\mathrm{n}=183(61 \%) / \mathrm{n}=173(58 \%))$ was substantially reduced $(\mathrm{P}<0.001)$ to $\mathrm{n}=43(14 \%) / \mathrm{n}=42$ $(14 \%)$ at the first study office visit when automated office blood pressure readings were taken. In contrast, digit preference in the control group with manual office blood pressure readings at the last routine office visit $(\mathrm{n}=128(51 \%) / \mathrm{n}=143(57 \%))$ was not significantly different from manual office blood pressure readings taken during the subsequent first study office visit $(\mathrm{n}=107(43 \%) / \mathrm{n}=124(50 \%))$. 
Table $2 \mid$ Mean (SD) blood pressure (BP) taken in physicians' office before and after enrolment into study and baseline mean awake ambulatory BP recorded between two office visits for patients randomised to intervention (automated office BP) and control (manual office BP) groups, with estimated mean differences $(95 \%$ confidence interval) between BP readings

\begin{tabular}{|c|c|c|}
\hline Measurement & Automated office BP group $(n=299)$ & Conventional manual office BP group $(n=249)$ \\
\hline Last routine manual office $\mathrm{BP}(\mathrm{mm} \mathrm{Hg})$ & $149.5(10.8) / 81.4(8.3)$ & $149.9(10.7) / 81.8(8.5)$ \\
\hline Office BP $(\mathrm{mm} \mathrm{Hg})$ after enrolment & $135.6(17.3) / 77.7(10.9)$ & $141.4(14.6) / 80.2(9.5)$ \\
\hline Difference from last routine office BP $(\mathrm{mm} \mathrm{Hg})$ & $-13.9(-11.8 \text { to }-16.1)^{\star \star \star} /-3.7(-2.5 \text { to }-4.8)^{\star \star \star}$ & $-8.5(-6.5 \text { to }-10.4)^{\star \star \star} /-1.6(-0.4 \text { to }-2.8)^{\star \star}$ \\
\hline Awake ambulatory BP $(\mathrm{mm} \mathrm{Hg})$ & $133.2(12.4) / 74.4(9.8)$ & $135.0(13.1) / 75.9(10.0)$ \\
\hline Difference from last routine office BP $(\mathrm{mm} \mathrm{Hg})$ & $-16.3(-14.5 \text { to }-18.1)^{\star \star \star} /-7.0(-5.8 \text { to } 8.1)^{\star \star \star}$ & $-14.9(-12.9 \text { to }-17.0)^{\star \star \star} /-5.9(-4.6 \text { to } 7.2)^{\star \star \star}$ \\
\hline Difference from post-enrolment office BP $(\mathrm{mm} \mathrm{Hg})$ & $-2.3(-0.31 \text { to }-4.3)^{\star} /-3.3(-2.2 \text { to }-4.4)^{\star \star \star}$ & $-6.5(-4.3 \text { to }-8.6)^{\star \star \star} /-4.3(-2.9 \text { to } 5.8)^{\star \star \star}$ \\
\hline $\begin{array}{l}{ }^{\star} \mathrm{P}=0.02 \\
{ }^{*} \mathrm{P}=0.01 \\
{ }^{* \star *} \mathrm{P}<0.001\end{array}$ & & \\
\hline
\end{tabular}

All six automated office blood pressure readings taken with the BpTRU device were recorded in 284 patients. The initial blood pressure with the health professionals still in the room (147 (SD 20)/82 (12) mm $\mathrm{Hg})$ was significantly $(\mathrm{P}<0.001)$ higher than all of the other blood pressure readings and the mean automated office blood pressure (table 4).

\section{DISCUSSION}

These initial findings in primary care patients with systolic hypertension are important in that they provide robust, internally and externally valid evidence to support the use of automated office blood pressure measurement in routine office practice. Manual office blood pressure readings taken before enrolment were significantly higher than the awake ambulatory blood pressure, which is considered to be a gold standard for defining blood pressure status. The routine manual office systolic blood pressure readings also correlated poorly with the awake ambulatory blood pressure. Replacement of manual office blood pressure measurement with automated office blood pressure virtually eliminated the difference between the routine manual office blood pressure and the awake ambulatory blood pressure (white coat effect). Automated office blood pressure readings also showed a stronger correlation with the awake ambulatory blood pressure than did manual readings.

A unique feature of the CAMBO trial is the inclusion of a control group of patients randomised to usual care with manual office blood pressure measurements. In CAMBO, the difference between the routine systolic/ diastolic manual and automated office blood pressure was $-13.9 /-3.7 \mathrm{~mm} \mathrm{Hg}$. Not all of this difference could be attributed to the intervention, as readings in the manual office blood pressure control group also decreased by $-8.5 /-1.6 \mathrm{~mm} \mathrm{Hg}$ after enrolment compared with before enrolment. The fall in blood pressure in the control group was probably due to several factors, including increased compliance with drug treatment after enrolment on the part of some patients, physicians and nurses reading blood pressure lower, and regression to the mean. Although changes in prescribed drugs were documented in the physicians' notes, patients were not specifically asked about compliance to avoid influencing their treatment. A change in technique for manual office blood pressure measurement on the part of the medical staff seems less likely, as readings taken on the first visit after enrolment were not any more accurate than the routine manual office blood pressure; digit preference was still present, and manual office blood pressure readings still correlated relatively poorly with the awake ambulatory blood pressure.

By subtracting the decrease in blood pressure in the control group from the decrease in the automated office blood pressure group, we can obtain the net reduction in blood pressure that can be attributed specifically to the automated office blood pressure intervention: $-5.4 /-2.1 \mathrm{~mm} \mathrm{Hg}$, which is still of considerable clinical importance. This value should be considered a minimum reduction in view of the likely changes in the patients' compliance with drug treatment and possible measurement bias based on the fall in blood pressure in the control group.

A recent report by Godwin et al provides further information on the effect that automated office blood pressure measurement might have on the white coat component of manual office blood pressure measurements. ${ }^{23}$ In their study, 654 treated

Table 3 |Coefficients of correlation between office systolic/diastolic blood pressure (BP) readings and mean awake ambulatory BP

\begin{tabular}{|c|c|c|}
\hline Measurements & Automated office BP group & Manual office BP gr \\
\hline Routine office BP before enrolment $v$ awake ambulatory BP & $r=0.10 / r=0.40$ & $r=0.04 / r=0.42$ \\
\hline First office BP after enrolment $v$ awake ambulatory BP & $r=0.34 / r=0.56^{*}$ & $r=0.22 / r=0.30$ \\
\hline $\begin{array}{l}\text { Estimated mean difference }(95 \% \mathrm{Cl}) \text { in } r \text { from before to after } \\
\text { enrolment }\end{array}$ & $0.24(0.12$ to 0.36$) \dagger / 0.16(0.07$ to 0.25$) \dagger$ & $0.18(0.02$ to 0.32$) \ddagger /-0.12(-$ \\
\hline
\end{tabular}


Intervention group

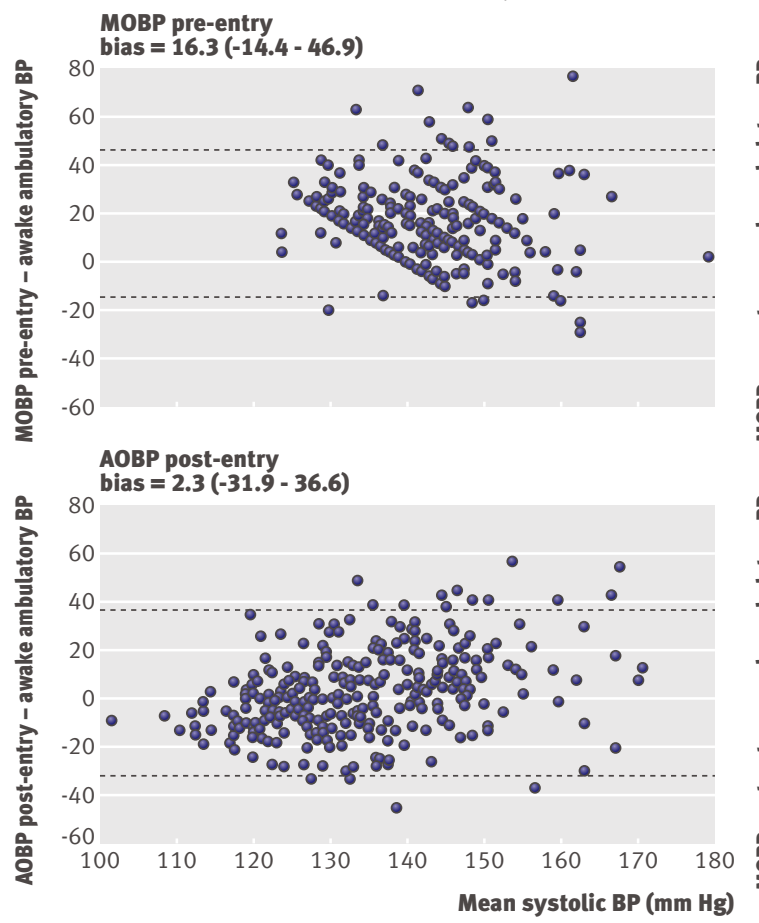

Control group
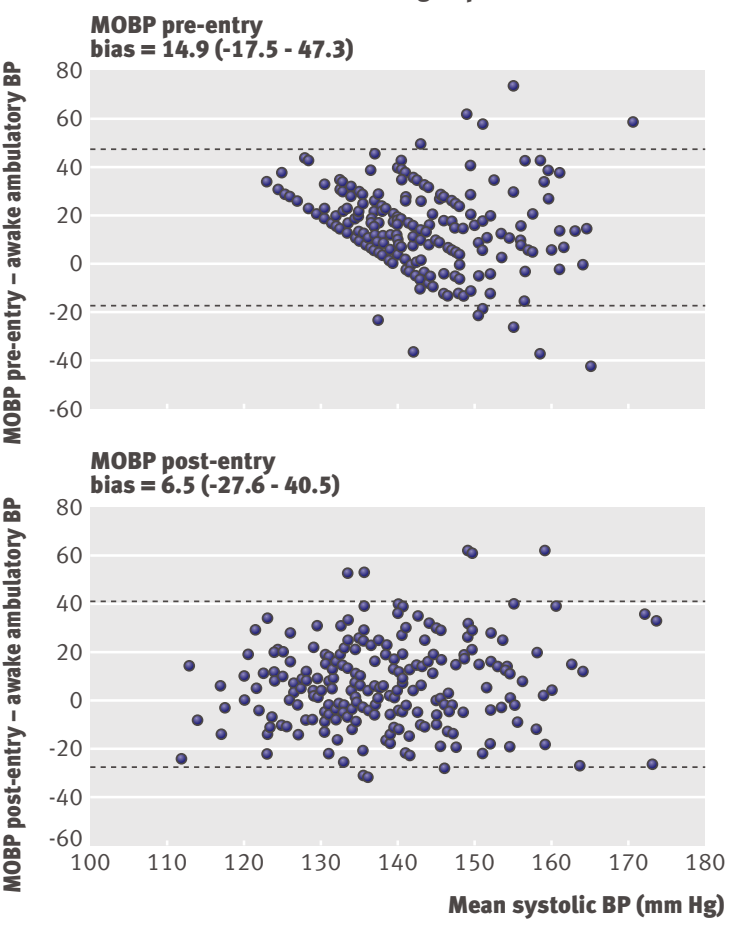

Fig 2 Mean of office blood pressure (BP) and awake ambulatory BP readings plotted against difference between these readings, using Bland-Altman format. ${ }^{21}$ Shows data for awake ambulatory BP versus routine manual office (MO) BP readings before enrolment (top panels), AOBP readings after enrolment (bottom left panel), and MOBP readings after enrolment (bottom right panel). Horizontal lines indicate two standard deviations around the mean difference. Values for estimated mean bias ( 2 SD) are shown

hypertensive patients had automated office blood pressure and 24 hour ambulatory blood pressure monitoring done immediately after providing written informed consent, leaving the patients no chance to increase their adherence to antihypertensive treatment. Under these circumstances, the routine manual office blood pressure of $148.5 / 82.5 \mathrm{~mm} \mathrm{Hg}$ was reduced to 139.2 / $79.8 \mathrm{~mm} \mathrm{Hg}$ when blood pressure recordings were taken with the BpTRU device. The corresponding mean awake ambulatory blood pressure was 140.9/ $79.6 \mathrm{~mm} \mathrm{Hg}$. Both the patients in this study and those in $\mathrm{CAMBO}$ were recruited from primary care practices in the community, and routine manual office blood pressure readings before enrolment were similar in the two studies.

Other populations have shown greater reductions in blood pressure when readings were taken with the BpTRU device. Routine manual office blood pressure in 254 untreated patients referred for 24 hour ambulatory blood pressure monitoring was reduced from 149.7/89.3 $\mathrm{mm} \mathrm{Hg}$ to $132.6 / 80.0 \mathrm{~mm} \mathrm{Hg}$ when automated office blood pressure was recorded in an ambulatory blood pressure monitoring unit. ${ }^{24}$ The corresponding awake ambulatory blood pressure was 135.3/81.0 mm Hg. The greater reduction in blood pressure in these patients was probably due to selection of patients, whereby physicians referred patients with suspected white coat hypertension for 24 hour ambulatory blood pressure monitoring. As these patients were untreated and routine manual office blood pressure readings were taken, little possibility exists that a change in patients' or physicians' behaviour could explain the lower automated office blood pressure values.

The introduction of automated office blood pressure into routine clinical practice could be thus expected to decrease systolic blood pressure readings by at least $5 \mathrm{~mm} \mathrm{Hg}$ and probably by 9 to $13 \mathrm{~mm} \mathrm{Hg}$ depending on the population of patients.

\section{Should manual office blood pressure measurement be abandoned?}

The mercury sphygmomanometer has been synonymous with blood pressure measurement for more than 100 years. Regardless of advances in alternative techniques for recording blood pressure, mercury seems destined to disappear from the workplace because of environmental concerns. Mercury is prohibited in at least two countries, ${ }^{25}$ and a recent report from the European Union suggests that a more widespread ban is imminent. ${ }^{26}$ The standard device for measuring blood pressure thus needs to be replaced, but the unknown factor is what comes next. Studies on white coat hypertension have identified a subset of about $25 \%$ of the hypertensive population who have clinically important increases in blood pressure when readings are taken in the treatment setting, especially by physicians. ${ }^{27}$ Even automated devices such as the BpTRU provoke a white coat response; readings are similar to manual office blood pressure if the observer 
Table $4 \mid$ Sequential mean (SD) blood pressure readings taken every two minutes in 284 patients with BpTRU device during first routine office visit after enrolment into study

\begin{tabular}{ll} 
Reading No & Automated office blood pressure $(\mathrm{mm} \mathrm{Hg})$ \\
1 & $147(20) / 82(12)^{\star}$ \\
\hline 2 & $140(20) / 79(12)$ \\
\hline 3 & $136(19) / 78(13)$ \\
\hline 5 & $134(18) / 77(12)$ \\
\hline 6 & $132(18) / 76(12)$ \\
\hline Mean 2-6 & $133(18) / 77(12)$ \\
\hline
\end{tabular}

${ }^{*} \mathrm{P}<0.001$ versus all subsequent readings.

remains present in the room when the reading is taken. ${ }^{17}$

Studies comparing manual with automated office blood pressure have clearly shown that the increase in blood pressure associated with conventional manual office measurement can be eliminated if the three basic tenets of automated office blood pressure are followed: the patient rests alone in a quiet room, and multiple readings are taken with a fully automated device. ${ }^{2}$ The simple presence of an observer seems to increase blood pressure, as marked decreases in automated office blood pressure are evident within two minutes of the observer leaving the room, as seen in CAMBO and in a previous study. ${ }^{17}$

The number of automated office blood pressure readings needed for the mean value to be equivalent to the awake ambulatory blood pressure depends on whether the patient rests before the first reading is taken..$^{28}$ If current guidelines for manual office blood pressure are followed and the patient is allowed to rest for at least five minutes, only two or three readings may be needed. However, when five readings are taken at one minute intervals with devices such as the BpTRU, no antecedent period of rest is needed and mean readings are still equivalent to the awake ambulatory blood pressure.$^{2930}$ Also, readings obtained with the BpTRU are similar when taken at one or two minute intervals. ${ }^{28}$

In countries such as Canada, physicians in primary care practice generally use several examining rooms for seeing patients. This arrangement is suitable for automated and manual office blood pressure measurement, even when the manual readings are taken according to guidelines with five minutes of rest before the first blood pressure measurement. When physicians have access to only one examining room, less time is available for optimal recording of a patient's blood pressure. Under these circumstances, both automated office blood pressure and a proper manual office blood pressure are generally not feasible. An essential feature of automated office blood pressure is the requirement that the patient should be seated in a quiet room for several minutes while readings are being taken. If only one or two readings are recorded without an antecedent period of rest, a white coat effect would probably affect the blood pressure value, as seen with the first two readings taken using the BpTRU device. ${ }^{17}$ The improved accuracy associated with automated office blood pressure is probably achieved by having the patient resting alone and by the use of an automated, validated device to take multiple, standardised readings.

Automated office blood pressure recorders need to be fully automated. In a recent study, ${ }^{30}$ the more expensive BpTRU device was replaced with validated, home blood pressure recorders, which patients activated five times at one minute intervals while alone in a quiet room. Mean blood pressure taken with the patient activated, automated recorders was about $5 \mathrm{~mm} \mathrm{Hg}$ higher than automated office blood pressure taken with the BpTRU, awake ambulatory blood pressure, and home blood pressure. If simply getting a patient to push a button every minute increases blood pressure, then the fact that manual office blood pressure readings in the presence of an observer are increased by at least $5 \mathrm{~mm} \mathrm{Hg}$ is not surprising.

Manual blood pressure also very much depends on the circumstances in which readings are taken. Pressure is higher when taken by physicians than by nurses, higher in treatment settings than in non-treatment settings, and lower when taken at home. ${ }^{3132}$ Manual office blood pressure can be 15-18 $\mathrm{mm} \mathrm{Hg}$ higher than the awake ambulatory blood pressure if recorded in routine clinical practice. ${ }^{1624}$ In contrast, automated office blood pressure and awake ambulatory blood pressure generally differ by less than $3 \mathrm{~mm} \mathrm{Hg}$, both in research settings and in clinical practice, as seen in CAMBO. ${ }^{2}$ Manual blood pressure also decreases if a patient moves from the physician's office to a non-treatment setting such as an ambulatory blood pressure monitoring unit, ${ }^{32}$ whereas automated office blood pressure does not fall. ${ }^{29}$

\section{Limitations of study}

The design of CAMBO is responsible for its major strengths and weaknesses. Despite the difficulties involved in doing a randomised controlled trial of blood pressure measurement in routine clinical practice, we could show that replacement of manual with automated office blood pressure could significantly reduce the white coat response associated with conventional office blood pressure measurement. By restricting the intervention solely to blood pressure measurement, we can generalise findings to hypertension management in most primary care practices, taking into consideration the fact that the patients in CAMBO were aged 45 and over, had predominantly systolic hypertension, did not have diabetes, and were free of any target organ damage. Further research is needed before the results of CAMBO can be extrapolated to other hypertensive populations.

CAMBO was designed to interfere as little as possible with routine management of patients. As a consequence, we could not provide instructions on proper technique for manual office blood pressure measurement, which would almost certainly have improved the quality of the readings. Moreover, research quality manual readings are generally not seen in routine 


\section{WHAT IS ALREADY KNOWN ON THIS TOPIC}

Increasing concern about the accuracy and reliability of manual blood pressure measurement in clinical practice has led to greater reliance on home and ambulatory blood pressure recordings using automated devices

The use of automated devices to measure blood pressure in the office setting with multiple readings taken while the patient rests alone in a quiet room overcomes many of the shortcomings of the manual technique

\section{WHAT THE STUDY ADDS}

Automated office blood pressure can be used in primary care practice to obtain valid readings without provoking the white coat response often seen with manual blood pressure measurement
Hypertension have also recommended the widespread use of home blood pressure monitoring. ${ }^{133}$ The American Heart Association report stated that "every hypertensive patient should purchase a home BP recording device." Although few hypertension experts have gone as far as recommending the complete abandonment of office blood pressure measurement, conventional manual office blood pressure readings are clearly widely viewed as providing suboptimal measures of an individual patient's blood pressure status.

Automated office blood pressure measurement provides an opportunity to continue recording blood pressure in the primary care setting, although it is not a replacement for home blood pressure or 24 hour ambulatory blood pressure monitoring. Identification of a close relation between readings taken with automated office blood pressure measurement and 24 hour ambulatory blood pressure monitoring suggests that future clinical outcome studies will confirm automated office blood pressure readings as being a better predictor of future cardiovascular events than are manual office blood pressure readings.

The initial findings from the CAMBO trial in patients with systolic hypertension and no serious comorbidities highlight the importance of recording blood pressure in the office with a fully automated device that takes multiple readings with the patient resting alone in the examining room. Further studies in other hypertensive populations are needed before automated office blood pressure can be recommended as a replacement for conventional manual blood pressure measurement.

We thank the primary care physicians and research assistants who participated in this study, which would not have been possible without their cooperation and strong commitment to the project (see web appendix for participating physicians).

Contributors: MGM is the principal investigator and was involved in all aspects from the development of the protocol to drafting the final manuscript and its revisions. MG participated in the design of the study, supervised the sites in St John's and Corner Brook, Newfoundland, and reviewed the manuscript and its revisions. MD was involved in the design of the study, supervised the sites in Montreal, and reviewed the manuscript and its revisions. AK was the statistician for the study, was involved in the initial sample size estimates and subsequent data analysis on completion of the trial, and advised on how to incorporate the data analysis into the final manuscript. SWT and JK contributed to the design of the study, review of the data, and preparation of the final manuscript. FCG supervised the sites in Belleville, Ontario, assisted with recruitment of patients, and reviewed the manuscript and its revisions. MGM is the guarantor

Funding: The study was supported by Grant ESA5745 from the Heart and Stroke Foundation of Ontario.

Competing interests: All authors have completed the Unified Competing Interest form at www.icmje.org/coi_disclosure.pdf (available on request from the corresponding author) and declare: no support from any organisation for the submitted work; no financial relationships with any organisations that might have an interest in the submitted work in the previous three years; no other relationships or activities that could appea to have influenced the submitted work.

Ethical approval: The study was approved by the Research Ethics Board of Sunnybrook Health Sciences Centre, Human Investigation Committee of Memorial University of Newfoundland, Institutional Review Board of McGill University Faculty of Medicine, St Mary's Hospital Center's

Research Review Office, and Comité D'éthique De La Recherche, Centre de Santé et de services sociaux de la Montagne.

Data sharing: No additional data available. 
1 Pickering TG, Hall JE, Appel LJ, Falkner BE, Graves J, Hill MN, et al. Recommendations for blood pressure measurement in humans and experimental animals part 1: blood pressure measurement in humans-a statement for professionals from the Subcommittee of Professional and Public Education of the American Heart Association Council on High Blood Pressure Research. Hypertension 2005;45:142-61.

2 Myers MG, Godwin M, Dawes M, Kiss A, Tobe S, Kaczorowski J. Measurement of blood pressure in the office-recognizing the problem and proposing the solution. Hypertension 2010;55:195-200.

3 Reeves RA. Does this patient have hypertension? How to measure blood pressure. JAMA 1995;273:1211-6.

4 Pickering TG, Gerin W, Schwartz JE, Spruill TM, Davidson KW. Should doctors still measure blood pressure? The missing patients with masked hypertension. J Hypertens 2008;26:2259-67.

5 Sala C, Santin A, Rescaldani M, Magrini F. How long shall the patient rest before clinic blood pressure measurement? Am J Hypertens 2006;19:713-7.

6 Giles TD, Egan P. Pay (adequately) for what works: the economic undervaluation of office and ambulatory blood pressure recordings. J Clin Hypertension 2008;10:257-9.

7 Parati G, Omboni S, Bilo G. Why is out-of-office blood pressure measurement needed? Hypertension 2009;54:181-7.

8 Verdecchia P, Angeli F, Mazzota G, Gentile G, Reboldi G. Home blood pressure measurements will not replace 24-hour ambulatory blood pressure monitoring. Hypertension 2009;54:188-95.

9 Stergiou GS, Siontis KCM, loannidis JPA. Home blood pressure as a cardiovascular outcome predictor: it's time to take this method seriously. Hypertension 2010;55:1301-3

10 Verdecchia P, Clement D, Fagard R, Palatini P, Parati G. Target-organ damage, morbidity and mortality. Blood Press Monit 1999;4:303-17.

11 Staessen JA, Byttebier G, Buntinx F, Celis H, O’Brien ET, Fagard R. Antihypertensive treatment based on conventional or ambulatory blood pressure measurement: a randomized controlled trial. JAMA 1997;278:1065-72.

12 Wright JM, Mattu GS, Perry TL Jr, Gelfer ME, Strange KD, Zorn A, et al. Validation of new algorithm for the BPM-100 electronic oscillometric office blood pressure monitor. Blood Press Monit 2001;6:161-5.

13 White WG, Anwar YA. Evaluation of the overall efficacy of the Omron office digital blood pressure HEM-907 monitor in adults. Blood Press Monit 2001;6:107-10.

14 Stergiou GS, Tzamouranis D, Protogerou A, Nasothimiou E, Kapralos C. Validation of Microlife Watch BP Office Professional device for office blood pressure measurement according to the international protocol. Blood Press Monit 2008;13:299-303.

15 Beckett L, Godwin M. The BpTRU automatic blood pressure monitor compared to 24-h ambulatory blood pressure monitoring in the assessment of blood pressure in patients with hypertension. $B M C$ Cardiovasc Disord 2005;5:18.

16 Myers MG, Valdivieso M, Kiss A. Use of automated office blood pressure measurement to reduce the white coat response. J Hypertens 2009;27:280-6.

17 Myers MG. Automated blood pressure measurement in routine clinical practice. Blood Press Monit 2006;11:59-62.

18 Ishikawa J, Nasothimiou E, Karpettas N, Feltheimer S, McDoniel S, Schwartz J, et al. Automatic office blood pressure measurement without doctors or nurses present is more predictive of ambulatory blood pressure than is office blood pressure by mercury sphygmomanometer. J Hypertens 2009;27(suppl 4):170S

19 Myers MG, Valdivieso M, Kiss A. Optimum frequency of automated blood pressure measurements using an automated sphygmomanometer. Blood Press Monit 2008;13:333-8

20 O'Brien E, Mee F, Atkins N, O'Malley K. Accuracy of the Spacelabs 90207 determined by the British Hypertension Society Protocol. Hypertens 1991;9(suppl 5):25-31S

21 Bland JM, Altman DG. Statistical methods for assessing agreement between two methods of clinical measurement. Lancet 1986;1:307-10

22 Donner A, Klar N. Design and analysis of cluster randomization trials in health research. Oxford University Press, 2000.

23 Godwin M, Birtwhistle R, Delva D, Lam M, Casson I, MacDonald S, et al. Manual and automated office measurements in relation to awake ambulatory blood pressure monitoring. Fam Pract 2011;28:110-7

24 Myers MG. A proposed algorithm for diagnosing hypertension using automated office blood pressure measurement. J Hypertens 2010;28:703-8

25 O'Brien E. Has conventional sphygmomanometry ended with the banning of mercury? Blood Press Monit 2002;7:37-40.

26 Scientific Committee on Emerging and Newly Identified Health Risks. Mercury sphygmomanometers in healthcare and the feasibility of alternatives. SCENIHR, 2009.

27 Pickering TG, James GD, Boddie C, Harshfield GA, Blank S, Laragh JH. How common is white coat hypertension? JAMA 1988,259:225-8.

28 Myers MG, Valdivieso M, Kiss A, Tobe SW. Comparison of two automated sphygmomanometers for use in the office setting. Blood Press Monit 2009;14:45-7.

29 Myers MG, Valdivieso M, Kiss A. Consistent relationship between automated office blood pressure recorded in different settings. Blood Press Monit 2009;14:108-11.

30 Myers MG, Valdivieso M, Chessman M, Kiss A. Can sphygmomanometers designed for self-measurement of blood pressure in the home be used in office practice? Blood Press Monit 2010;15:300-4.

31 Head GA, Mihailidou AS, Duggan KA, Beilin LI, Berry N, Brown MA, et al. Definition of ambulatory blood pressure targets for diagnosis and treatment of hypertension in relation to clinic blood pressure: prospective cohort study. BMJ 2010;340:1104-11.

32 Myers MG, Valdivieso MA. Use of an automated blood pressure recording device, the BPTRU, to reduce the "white coat effect" in routine practice. Am J Hypertens 2003;16:494-7.

33 Mancia G, De Backer G, Dominiczak A, Cifkova R, Fagard R, Germano G, et al. 2007 guidelines for the management of arterial hypertension: the Task Force for the Management of Arterial Hypertension of the European Society of Hypertension (ESH) and of the European Society of Cardiology (ESC). J Hypertens 2007;25:1105-87

34 O'Brien E. Ambulatory blood pressure measurement: the case for implementation in primary care. Hypertension 2008;51:1435-41.

Accepted: 28 November 2010 